\title{
ÍNDICES PRODUTIVOS DE ABELHAS NATIVAS ASSOCIADOS À CONDIÇÕES AMBIENTAIS EM ARAPIUNS, PARÁ
}

\author{
Adcléia Pereira Pires'; Aline Pacheco²; Lucieta Guerreiro Martorano ${ }^{3}$; Alanna do Socorro Lima da Silva²; Ana \\ Paula da Silva Viana1; Marcelo Costa Diniz'; Andria Tavarez Galvão1; José Reinaldo da Silva Cabral de \\ Moraes ${ }^{4}$. \\ ${ }^{1}$ Alunos de Graduação, Universidade Federal do Oeste do Pará (UFOPA), Instituto de \\ Biodiversidade e Floresta (IBF), Santarém, Pará, Brasil, piresadcleia@gmail.com; anastm.paula@gmail.com; \\ marcelodinizag@gmail.com; andriataveres@hotmail.com \\ 2 Professora Adjunta, UFOPA/IBF, Santarém, Pará, Brasil, alinepacheco@outlook.com \\ 3 Pesquisadora Embrapa Amazônia Oriental/NAPTdo Médio Amazonas, Santarém, Pará, Brasil, \\ lucieta.martorano@embrapa.br \\ ${ }^{4}$ Aluno de mestrando do Programa de Pós-Graduação em Agronomia (Produção Vegetal), da \\ Universidade Estadual Paulista (UNESP), Jaboticabal, São Paulo, Brasil, reinaldojmoraes@gmail.com
}

RESUMO: Os animais quando submetidos a condições ambientais fora da zona de termoneutralidade desenvolvem estratégias de sobrevivência que podem comprometer suas funcionalidades, principalmente na produção de alimentos às populações. Objetivouse identificar índices produtivos de abelhas nativas associados às condiç̃̃es ambientais em colônias de Melipona interrupta criadas racionalmente na comunidade Coroca, Rio Arapiuns. Foi realizado a bionomia de 15 colmeias durante um ano e para diagnosticar as condições em colônias dessas abelhas utilizou-se um termógrafo infravermelho. As chuvas na região concentram-se entre os meses de janeiro a junho, sendo as maiores ofertas pluviais nos meses de março e abril, contabilizando-se 280 mm e 270 mm, respectivamente. No período menos chuvoso as temperaturas médias do ar encontravam-se mais elevadas, sendo esse período o mais propício à floração das espécies nativas, aumentando a oferta de alimentos às abelhas e consequentemente aumenta a produção de mel. Os padrões térmicos nos alvos indicaram que a entrada dos potes de mel estava com as temperaturas mais elevadas, atingindo $43,0^{\circ} \mathrm{C}$ com amplitude térmica de $3,4^{\circ} \mathrm{C}$. Na parede das caixas e nos espaços entre os potes de mel foram detectadas temperaturas semelhantes de $34,0{ }^{\circ} \mathrm{C}$ que apontavam indícios de estresse térmico, conforme resultados disponíveis na literatura. Como as abelhas utilizam estratégias de termorregulação notou-se reduzida oscilação da temperatura nos potes de mel, explicada pela capacidade da cera em funcionar como isolante e conservante para as colmeias. Conclui-se que a produtividade de mel no período das mais elevadas temperaturas reforça a adaptabilidade dessa espécie às condições climáticas da região.

PALAVRAS-CHAVE: Melipona interrupta, Meliponicultura, Serviços Ecossistêmicos, Termorregulação. 


\title{
PRODUCTIVE INDICES OF NATIVE BEES ASSOCIATED TO ENVIRONMENTAL CONDITIONS AS MEASURED BY INFRARED THERMOGRAPHY IN ARAPIUNS, PARÁ
}

\begin{abstract}
When animals are subjected to environmental conditions outside of the thermoneutral zone they develop survival strategies that can compromise their metabolic functions, principally with respect to food production. The objective of this study was to identify productive indices of native bees associated to environmental conditions in colonies of Melipona interrupta raised through beekeeping techniques in the Coroca community in the region of the Arapiuns River. In order to diagnose environmental conditions in the 15 bee colonies, infrared thermography was used. Rainfall in the region is concentrated between the months of January and June with the highest rainfall occurring in March and April (280 mm and $270 \mathrm{~mm}$, respectively). In the rainy season average air temperature was higher than in the dry season, and this period is when the majority of native plant species flower, thus increasing the availability of food sources for bees and consequently the production of honey. Analysis of thermal patterns revealed that the area near the entry to the honey pots had higher temperatures, reaching $43.0^{\circ} \mathrm{C}$ with a thermal amplitude of $3.4{ }^{\circ} \mathrm{C}$. At the walls of the boxes and in the spaces between the honey pots temperatures of $34.0{ }^{\circ} \mathrm{C}$ were detected which signals that the colony is experiencing thermal stress, in agreement with results from the literature from other studies on this subject. Since these bees use thermal-regulation strategies, a notable oscillation of temperature was detected near the honey pots, which can be explained by the capacity of wax to function as a material that provides isolation from heat and water. We conclude that the continued production of honey during the period of higher air temperatures reinforces the concept that these bees possess the capacity to adapt to changing climate conditions in this region.
\end{abstract}

KEYWORDS: Ecosystem Services, Melipona interrupta, Meliponicultura, Thermal regulation.

\section{ÍNDICES PRODUCTIVOS DE ABEJAS NATIVAS ASOCIADOS A CONDICIONES AMBIENTALES EN ARAPIUNS, PARÁ}

RESUMEN: Los animales cuando sometidos a condiciones ambientales fuera de la zona de termo neutralidad desarrollan estrategias de supervivencia que pueden comprometer sus funciones, principalmente en producción de alimentos a las poblaciones. Se objetivó identificar índices productivos de abejas nativas asociados a condiciones ambientales en colonias de Melipona interrupta criadas racionalmente en la comunidad Coroca, Rio Arapiuns. Fue realizada la bionomia de 15 colmenas durante un año y para diagnosticar las condiciones en colonias de esas abejas se utilizó un termógrafo infrarrojo. Las lluvias en la región se concentran entre los meses de Enero a Junio, siendo las mayores ofertas 
pluviales en los meses de Marzo y Abril, contabilizándose 280 mm y 270 mm, respectivamente. En el período menos lluvioso las temperaturas medias se encontraban más elevadas, siendo ese período el más propicio para floración de especies nativas, aumentando la oferta de alimentos a las abejas y consecuentemente aumentando la producción de miel. Los patrones térmicos en los blancos indicaron que la entrada de los potes de miel estaba con las temperaturas más elevadas, alcanzando 43,0 ${ }^{\circ} \mathrm{C}$ con amplitud térmica de $3,4^{\circ} \mathrm{C}$. En la pared de las cajas y en los espacios entre los potes de miel fueron detectadas temperaturas semejantes de $34,0{ }^{\circ} \mathrm{C}$ que apuntaban indicios de estrés térmico, conforme resultados disponibles en la literatura. Como las abejas utilizan estrategias de termorregulação se notó reducida oscilación de la temperatura en los potes de miel, explicada por la capacidad de la cera en funcionar como aislante y conservante para las colmenas. Se concluye que la productividad de miel en el período de temperaturas más elevadas refuerza la adaptabilidad de esta especie a las condiciones climáticas de la región.

PALABRAS CLAVE: Melipona interrupta, Meliponicultura, Servicios Ecosistémicos, Termorregulación.

\section{INTRODUÇÃO}

As abelhas nativas da tribo Meliponini, conhecidas como abelhas sem ferrão foram classificadas por Camargo e Pedro (2012), como insetos sociais, sendo o gênero Melipona. Apresentam importância ecológica e econômica, pela especificidade em polinizar plantas nativas e cultivadas, além da qualidade do mel produzido por esses insetos (NATES-PARRA; ROSSO-LONDOÑO, 2013; KERR et al., 1966).

Os registros de ocorrência natural concentram-se na faixa Neotropical, contabilizando-se o maior número de espécies, sendo que no Brasil as identificações atingem aproximadamente 200 espécies de meliponínios (VENTURIERI et al., 2003; SOUZA et al., 2009; CAMARGO; PEDRO 2012). Dentro dos meliponínios são encontrados três gêneros: Melipona, Trigona e Trigonini. Como o gênero Melipona é dominante no Brasil, pela biodiversidade de padrões florísticos no país, o cultivo dessas abelhas denomina-se meliponicultura (NOGUEIRA-NETO, 1997). Essas atividades de criação racional de abelhas sem ferrão são realizadas em caixas de 
madeira e, instaladas em ambientes denominados meliponários.

Utilizando-se técnicas de manejo racional o objetivo principal é produzir mel, pólen e própolis. Entre as diferentes aplicações, os produtos derivados do material produzido pelas abelhas podem receber diferentes usos, inclusive na dieta de comunidades de povos tradicionais pelo alto valor nutritivo (CARVALHOZILSE et al., 2005; BARBOSA-COSTA et al., 2011), bem como agregação de valor sócio-econômico ao produtor rural pelo trabalho em grupo e venda dos produtos (VENTURIERI et al., 2003; CARVALHOZILSE; NUNES-SILVA, 2012).

Vale ressaltar os inúmeros benefícios da meliponicultura, principalmente pelos serviços ecossistêmicos prestados pelas abelhas (KLEIN et al., 2007) como polinizadoras de aproximadamente de 90\% da flora nativa (KERR, 1997), regulando a produção de sementes férteis e frutos e provendo a conservação ambiental pela interconectividade entre a fauna, a flora e o homem. O desflorestamento, crescimento populacional, dentre outros fatores apresentam ameaças a manutenção das abelhas nos ecossistemas terrestres (IMPERATRIZ-FONSECA; NUNES-SILVA, 2010; CARVALHO-ZILSE; ALMEIDA, 2013).

As abelhas vêm sendo ameaçadas pelo desenvolvimento desordenado, degradação e fragmentação de florestas, uso indiscriminado de agroquímicos, presença de espécies invasoras em áreas cultivadas, desiquilíbrio no aumento de inimigos naturais e, pelas ameaças com a elevação térmica em cenários de mudanças climáticas (BROWN; PAXTON, 2009). As mudanças climáticas alteram o comportamento dos seres vivos pela distinta tolerância e diferentes formas de reação às condições ambientais (SOMMER et al., 2010).

Em se tratando de abelhas nativas a termorregulação dentro dos ninhos é fundamental para a sobrevivência das colônias (HEINRICH, 1993); ROLDÃO, 2011). Em dias com temperaturas elevadas, as abelhas perdem eficiência com gastos energéticos, ou seja, em dias quentes $\left(>30^{\circ} \mathrm{C}\right)$ fornecendo ventilação dentro das colônias e, dias fria $\left(<26^{\circ} \mathrm{C}\right)$, construindo invólucros de cera para 
armazenar calor, garantindo assim a regulação térmica nas colmeias (BECKER, 2014). Para Roldão (2011) as temperaturas inadequadas comprometem o desenvolvimento das crias e, características morfofisiológicas causando má formação e mortalidade das crias.

Nesse contexto, a utilização de respostas usando termografia infravermelho (TIV) apresenta-se como alternativa não invasiva na obtenção de temperaturas de diferentes alvos permitindo avaliar a fisiologia dos animais nas áreas de produção, evitando estresse no manejo, pela redução de contato humano (BUSTOS MAC-LEAN, 2014).

Assim, o objetivo com este trabalho foi identificar índices produtivos de abelhas nativas associados às condições ambientais, diagnosticadas por termografia infravermelho em colônias de Melipona interrupta criadas racionalmente na comunidade Coroca, Rio Arapiuns, Pará.

\section{MATERIAL E MÉTODOS}

A área de estudo está localizada no assentamento agroextrativista, na comunidade Coroca, no Rio Arapiuns, município de Santarém, Pará, nas coordenadas geográficas latitude $02^{\circ} 25^{\prime} 10.4^{\prime \prime} \mathrm{S}$ e longitude $55^{\circ} 12^{\prime} 44.2^{\prime \prime} \mathrm{W}$. O período analisado neste trabalho compreende ao início de outubro de 2016 a início de outubro de 2017, sendo as coletas realizadas mensalmente, e cada coleta foi realizada na primeira semana de cada mês. Na Figura 1, está representado o mapa de localização onde foram realizadas as coletas de dados.

A cada mês foi realizado a bionomia de quinze colônias de abelhas nativas da espécie Melipona interrupta (Jandaíra). As colônias pertenciam a um meliponário de produção familiar, e eram mantidas em caixas modelo INPA com medidas de $20 \times 20 \times 8 \mathrm{~cm}$. 
Figura 1. Mapa da comunidade agroextrativista Coroca, Rio Arapiuns, Pará.

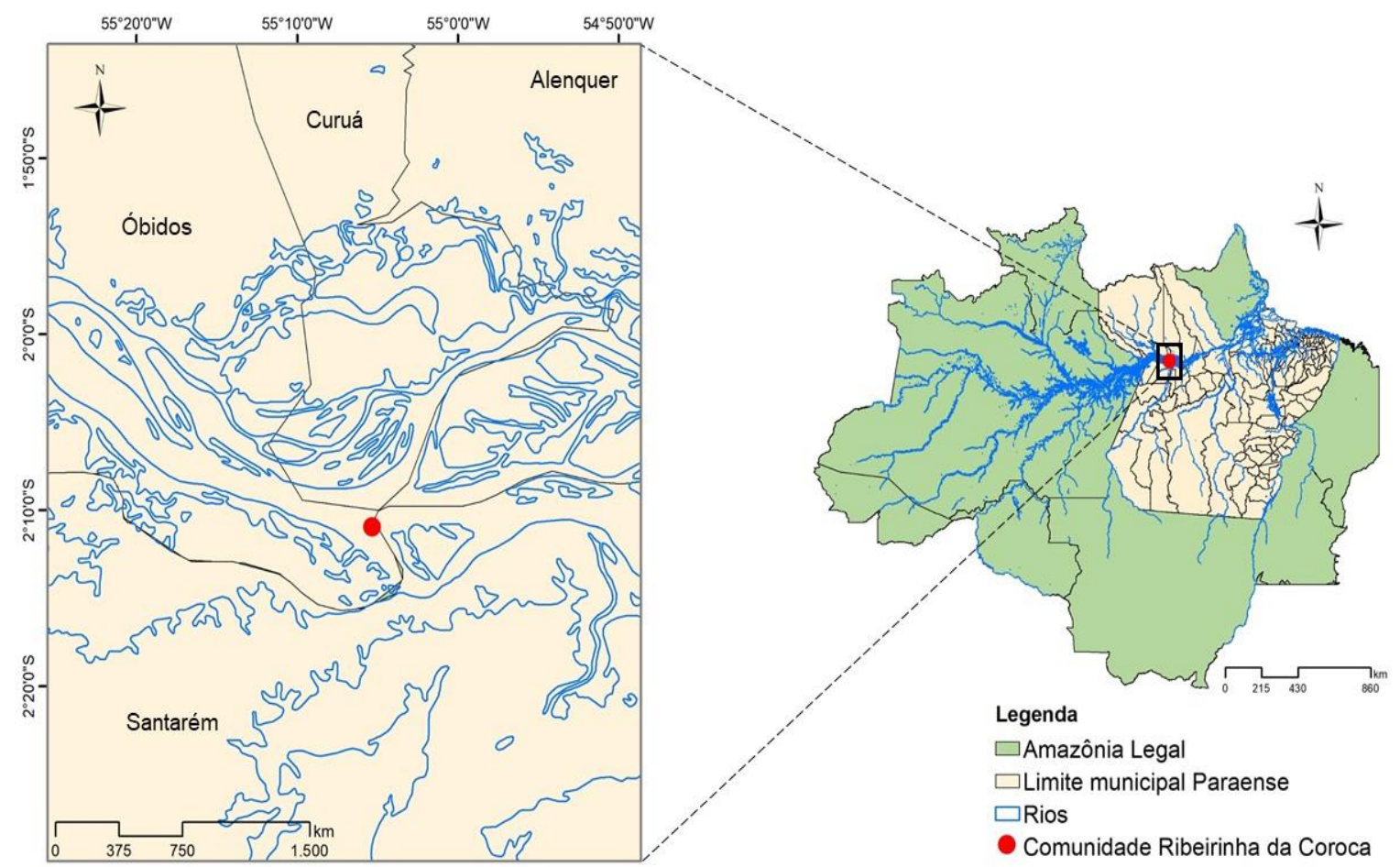

Para realizar a bionomia as colônias (VPM) foi mensurado fazendo-se a de abelha nativa foram abertas, e sucção do conteúdo dos potes, com realizou-se as seguintes medidas: largura dos potes de mel (LPM); profundidade dos potes de mel (PPM); largura dos potes de pólen (LPP); profundidade dos potes de pólen (PPP) com auxílio do paquímetro. Número total dos potes de mel (NPM); número total dos potes de pólen (NPP); peso da massa do pólen (PMP), o pólen foi retirado com o auxílio de uma espátula e pesado em balança digital. O volume dos potes de mel auxilio de seringas descartáveis, graduadas de $20 \mathrm{ml}$ (Figura 2).

Para avaliação das características produtivas, foram computados a largura, a profundidade e o volume dos potes de mel de ( $n=15 /$ colônia) e de pólen ( $n=$ 15/colônia). Para calcular o volume total de mel (VTM) por colmeia, foi realizada a contagem de potes operculados, multiplicando-se o número obtido pelo volume de potes com mel encontrados a 
cada coleta de campo. A estimativa da quantidade total de pólen (QTP) por colmeia foi realizada multiplicando-se o número de potes operculados pela média geral do peso da massa do pólen dos potes.

Foram extraídos dados climáticos para subsidiar as análises do ambiente na área de estudo, referentes ao período de 1990 a 2010, provenientes do modelo climático do ECMWF (modelo global de clima do Centro Europeu de Previsões de Médio Prazo). Assim sendo, fez-se uma análise sobre a temperatura do ar média e a quantidade de chuva mensal na área de estudo.

Para avaliar as condições térmicas nas colmeias, foram realizadas coletas de imagens usando termografia infravermelho. Como o período de redução das chuvas ocorre a partir do mês de julho, fez-se a coleta termográfica durante as coletas de agosto de 2017, ou seja, considerando-se o início da florada, momento que as abelhas operárias intensificam as coletas de pólen e néctar (forrageamento). As imagens termográficas foram obtidas em colmeias fechadas e abertas, utilizando uma a câmera Flir T650sc, número de série 55906998, lente Fol 25 mm e resolução IR 640 x 480, sendo as imagens tratadas no Flir Tools versão 6.3, onde foram avaliadas as variações térmicas nos diferentes alvos dentro e fora das colmeias e nas caixas contendo os ninhos.

Os dados foram analisados através do uso do Programa computacional SAS Statistical Analysis System. Foi utilizado delineamento inteiramente casualizado. Para a comparação entre os tratamentos (período mais chuvoso e período menos chuvoso), foram realizadas análises de variância e, para a comparação das médias foi utilizado o teste de Tukey. Foram calculadas as correlações simples entre características produtivas (Proc. CORR, SAS, 1999) associadas às variáveis biométricas. 
Figura 2. Imagens fotográficas demonstrando o processo de coleta: A. Meliponário; B. Formas dos potes de mel; C. Discos de crias maduras; D. Coletas com seringa do volume nos potes (Fotos: Primeira autora).
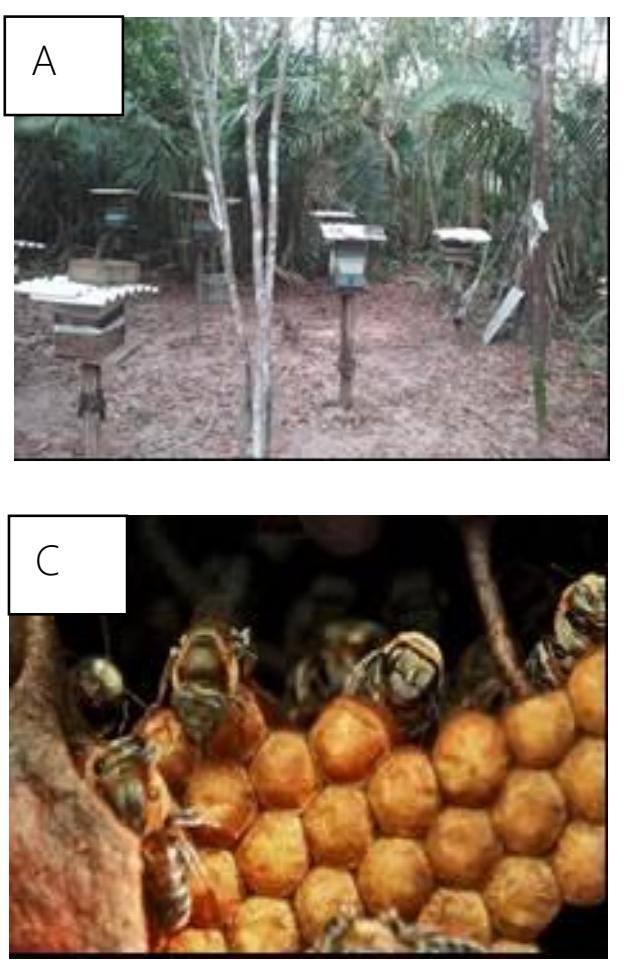

\section{RESULTADOS E DISCUSSÃO}

Observa-se na Figura 3 que as chuvas na região concentram-se entre os meses de janeiro a junho, sendo as maiores ofertas pluviais nos meses de março e abril, contabilizando-se $280 \mathrm{~mm}$ e 270 $\mathrm{mm}$, respectivamente. Nesses dois meses mais chuvosos são registradas as menores temperaturas médias que ficam em $25^{\circ} \mathrm{C}$. Outubro é o mês menos
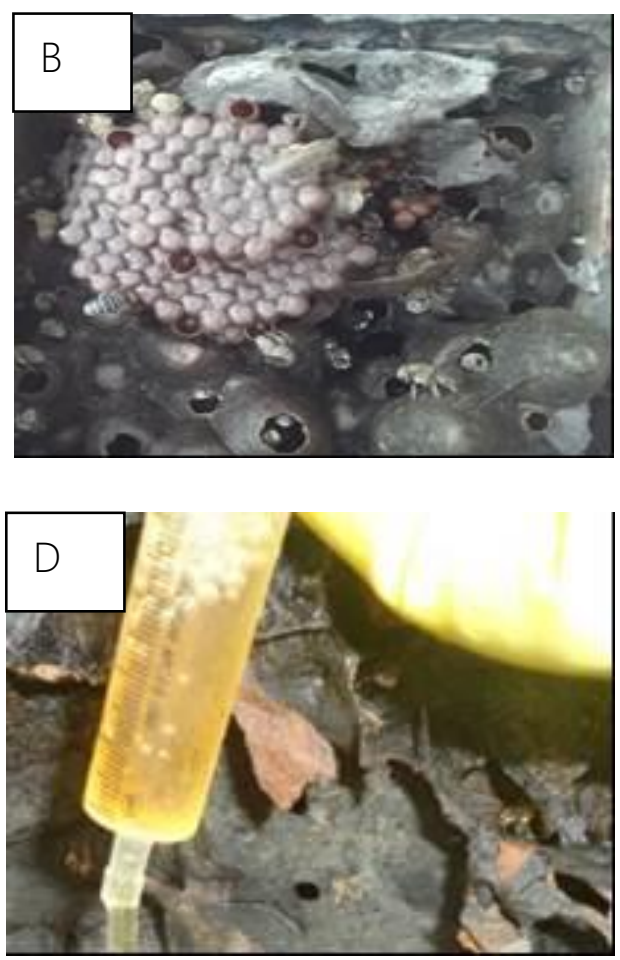

chuvoso com valores inferiores a 100 $\mathrm{mm}$, sendo o mês mais quente atingindo os $28^{\circ} \mathrm{C}$ de temperatura média do ar. No período menos chuvoso as temperaturas médias do ar encontram-se mais elevadas, sendo esse período o mais propício a floração das espécies nativas, aumentando a oferta de alimentos às abelhas e consequentemente aumenta a produção de mel. 
Figura 3. Dinâmica mensal da temperatura média do $\operatorname{ar}\left({ }^{\circ} \mathrm{C}\right)$ e da precipitação pluvial (mm), no período de 1990 a 2010 extraído do modelo climático do ECMWF.

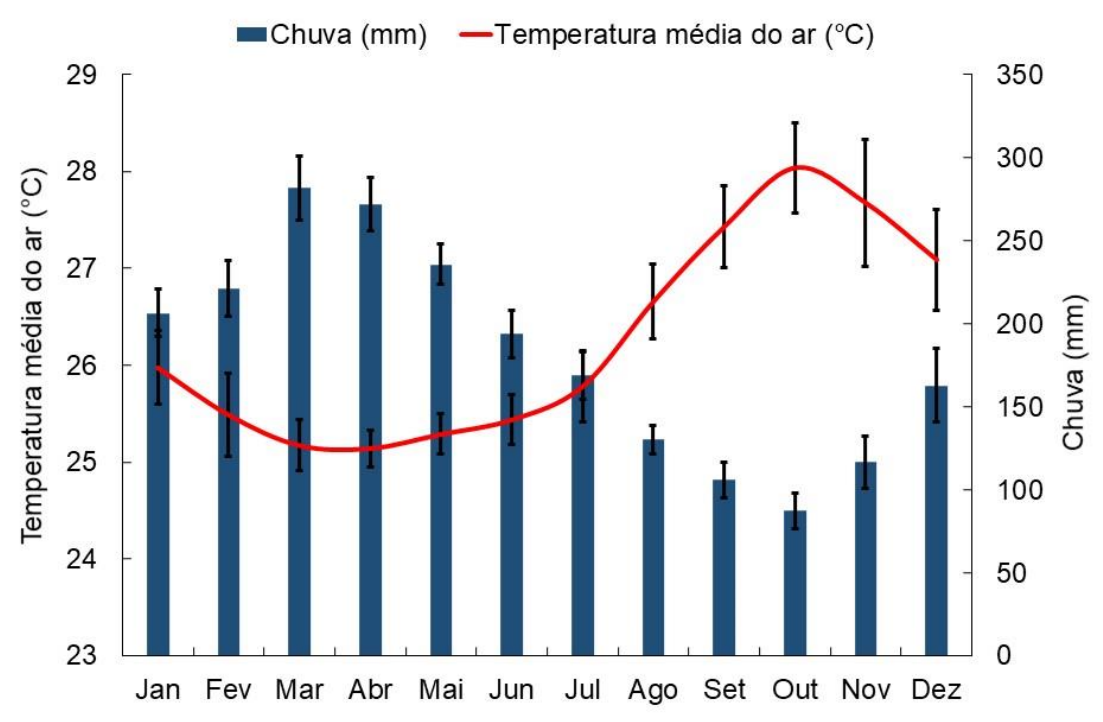

As médias e os respectivos desvios padrão das características produtivas de colônias de Melipona interrupta de acordo com o período do ano estão descritos na Tabela 1. A característica LPP (largura dos potes de pólen) não apresentou variação ao longo do ano, indicando que essa variável resposta se mantem em uma faixa de 2,32 $\pm 0,11$ a

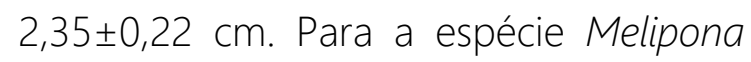
asilvai Sousa et al. (2008) encontraram valores inferiores de 2,03 $\pm 0,31 \mathrm{~cm}$.

As características LPM (largura dos potes de mel), VPM (volume dos potes de mel), PMP (peso da massa de pólen),
NPP e NPM (número de potes de pólen e de mel), VTM (volume total de mel) e QTP (quantidade total de pólen) apresentaram valores mais elevados no período menos chuvoso (outubro a dezembro), que coincide com o período de maior floração na região. As características APM e APP apresentaram valor superior no período mais chuvoso

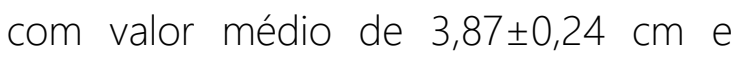
$4,84 \pm 0,28$, respectivamente (Tabela 1).

Dias et al. (2008) em estudos realizados com abelhas Jandaíra (Melipona subtinida), encontraram valor médio de 55,43 para número de potes 
de mel (NPM) em colônias alimentadas artificialmente. Alves (2010) em colônias de M. scutellaris relatou média para NPM de 14,96. Estes valores relatados são superiores aos encontrados para NPM tanto no período mais chuvoso quanto no menos chuvoso $(5,05 \pm 1,14$ e $11,96 \pm 7,14)$. Deve se considerar que cada espécie de meliponineos constrói potes de tamanhos diferentes e que fatores externos influenciam (PEREIRA et al., 2011).

Na Tabela 2 as médias de bionomia foram analisadas por ano e verifica-se que a média para volume total de mel (VTM), em 2016 foi de 227,48 ml e para QTP foi de 168,33g. No entanto neste ano foram realizadas coletas apenas no período de outubro a dezembro, que coincide com o período de menor oferta pluvial e temperaturas elevadas.

Os resultados evidenciam que as características produtivas relacionadas às épocas mais chuvosas e menos chuvosas tiveram diferenças significativas (Tabela 1), sendo os valores superiores quando é reduzida a oferta das chuvas, ou seja, as plantas intensificam a florada para perpetuarem as espécies visitadas pelas abelhas. Por outro lado, no período mais chuvoso as abelhas ampliam a altura dos potes de mel (APM) e altura dos potes de pólen.

De acordo Alves (2010) a produção de mel está intrinsecamente ligada ao número de potes de mel e, consequentemente esta relacionada a quantidade de cera produzida pelas abelhas operárias que utilizam mel e resina na construção desses potes, destacando-se ainda que, quando a LPM e APM apresentam medidas expressivas, ocorre redução do consumo de mel por operárias para produção de potes.

A quantidade de produção de mel além de estar relacionada com a disponibilidade de florada no raio de vôo necessita de expressivo número de operárias campeiras durante o período de coleta de néctar. Portanto, há necessidade de manejo suplementando artificialmente para fortalecimento das colônias, para assim maximizar o armazenamento no período de safra (EVANGELISTA-RODRIGUES et al., 2008). 
Tabela 1. Média, desvio padrão (DP) e coeficiente de variação das características produtivas avaliadas em colônias de Melipona interrupta, na comunidade Coroca, Arapiuns/PA, em dois períodos (mais chuvoso e menos chuvoso)

\begin{tabular}{|c|c|c|c|}
\hline \multirow{3}{*}{$\begin{array}{c}\text { Características } \\
\text { Biométricas }\end{array}$} & \multicolumn{3}{|c|}{ Período } \\
\hline & \multirow{2}{*}{$\begin{array}{l}\text { Mais chuvoso } \\
\text { (jan a jun) } \\
\text { Média } \pm \mathrm{DP}\end{array}$} & \multicolumn{2}{|c|}{$\begin{array}{c}\text { Menos chuvoso } \\
\text { (jul a dez) }\end{array}$} \\
\hline & & Média $\pm D P$ & CV\% \\
\hline LPM (cm) & $2,22 \pm 0,15^{B}$ & $2,28 \pm 0,25^{A}$ & 9,68 \\
\hline VPM (mL) & $9,61 \pm 1,26^{B}$ & $12,38 \pm 3,22^{A}$ & 19,88 \\
\hline APM $(\mathrm{cm})$ & $3,87 \pm 0,24^{A}$ & $3,60 \pm 0,50^{B}$ & 9,63 \\
\hline PMP (g) & $13,99 \pm 2,67^{B}$ & $16,01 \pm 2,36^{A}$ & 16,10 \\
\hline LPP (cm) & $2,32 \pm 0,11^{A}$ & $2,35 \pm 0,22^{A}$ & 7,79 \\
\hline APP (cm) & $4,84 \pm 0,28^{A}$ & $4,25 \pm 0,68^{B}$ & 10,15 \\
\hline NPM (unidade) & $5,05 \pm 1,14^{B}$ & $11,96 \pm 7,14^{\mathrm{A}}$ & 39,87 \\
\hline NPP (unidade) & $5,76 \pm 2,48^{B}$ & $8,51 \pm 2,27^{A}$ & 30,16 \\
\hline VTM $(m L)$ & $49,05 \pm 14,50^{B}$ & $158,58 \pm 109,11^{\mathrm{A}}$ & 56,72 \\
\hline QTP $(g)$ & $84,76 \pm 48,35^{B}$ & $138,36 \pm 48,25^{A}$ & 39,02 \\
\hline
\end{tabular}

LPM: largura dos potes de mel; VPM: volume dos potes de mel, APM: altura dos potes de mel; PMP: peso da massa do pólen; LPP: largura dos potes de pólen; APP: altura dos potes de pólen; NPM: número de potes de mel; NPP: número dos potes de pólen; VTM: volume total de mel; QTP: quantidade total de pólen. Médias seguidas por letras diferentes na mesma linha diferem estatisticamente entre si pelo teste Tukey a $1 \%$. CV = coeficiente de variação.

Tabela 2. Média e desvio padrão (DP) das características produtivas avaliadas em colônias de Melipona interrupta, na comunidade Coroca, Arapiuns/PA, nos anos 2016 e 2017

\begin{tabular}{lccc}
\hline \multirow{2}{*}{$\begin{array}{c}\text { Características } \\
\text { Biométricas }\end{array}$} & Média \pm DP & 2016 (jan a out) & \\
\cline { 2 - 4 } LPM (cm) & $2,30 \pm 0,25^{\mathrm{A}}$ & Média $\pm \mathrm{DP}$ & $\mathrm{CV} \%$ \\
VPM (mL) & $12,62 \pm 2,18^{\mathrm{A}}$ & $2,24 \pm 0,20^{\mathrm{A}}$ & 9,71 \\
APM (cm) & $3,34 \pm 0,40^{\mathrm{B}}$ & $10,65 \pm 2,90^{\mathrm{B}}$ & 24,80 \\
PMP (g) & $16,87 \pm 2,23^{\mathrm{A}}$ & $3,84 \pm 0,35^{\mathrm{A}}$ & 9,84 \\
LPP (cm) & $2,33 \pm 0,24^{\mathrm{A}}$ & $14,53 \pm 2,59^{\mathrm{B}}$ & 16,70 \\
APP (cm) & $16,87 \pm 0,56^{\mathrm{B}}$ & $2,34 \pm 0,16^{\mathrm{A}}$ & 7,82 \\
NPM (unidade) & $18,00 \pm 5,09^{\mathrm{A}}$ & $14,53 \pm 0,49^{\mathrm{A}}$ & 11,33 \\
NPP (unidade) & $9,93 \pm 1,71^{\mathrm{A}}$ & $6,00 \pm 3,29^{\mathrm{B}}$ & 43,14 \\
VTM (mL) & $227,48 \pm 76,61^{\mathrm{A}}$ & $6,44 \pm 2,46^{\mathrm{B}}$ & 31,91 \\
QTP (g) & $168,33 \pm 38,86^{\mathrm{A}}$ & $72,19 \pm 70,71^{\mathrm{B}}$ & 66,75 \\
\hline
\end{tabular}

LPM: largura dos potes de mel; VPM: volume dos potes de mel, APM: altura dos potes de mel; PPM: peso da massa do pólen; LPP: largura dos potes de pólen; APP: altura dos de pólen; NPM: número de potes de mel; NPP: número dos potes de pólen; VTM: volume total de mel; QTP: quantidade total de pólen. Médias seguidas por letras diferentes na mesma linha diferem estatisticamente entre si pelo teste Tukey a $1 \%$. CV= coeficiente de variação. 
Na Tabela 3 estão as correlações entre as características produtivas. O VPM (volume de potes de mel) apresentou alta correlação com NPM $(r=0,59)$, VTM $(r=0,79), \operatorname{NPP}(r=0,52)$ e PMP $(r=0,43)$ e QTP $(r=0,55)$, reforçando que a espécie estudada e as condições ambientais influenciam nas produções de mel e de pólen, ou seja, existe correlação favorável entre produção de mel e pólen. Por outro lado, as variáveis altura dos potes de mel (APM) e altura de potes de pólen (APP) apresentaram correlações negativa com as demais características produtivas de mel (VPM, NPM, VTM) e de pólen (NPP, PMP, QTP). A largura dos potes de mel (LPM) e a largura dos potes de pólen (LPP) apresentaram correlações positivas, sugerindo que a quantidade de mel está diretamente relacionada à largura dos potes.

Tabela 3. Correlação entre as características biométricas e produtivas em abelhas (Melipona interrupta).

\begin{tabular}{llllllllll}
\hline & VPM & APM & LPM & LPP & APP & PMP & NPM & NPP & VTM \\
\hline VPM & - & & & & & & & & \\
APM & -0.21 & - & & & & & & & \\
LPM & 0.26 & 0.27 & - & & & & & & \\
LPP & 0.20 & 0.05 & 0.49 & - & & & & & \\
APP & -0.56 & 0.53 & -0.13 & NS & - & & & & \\
PMP & 0.43 & -0.25 & 0.04 & 0.05 & -0.36 & - & & & \\
NPM & 0.59 & -0.54 & 0.14 & 0.08 & -0.68 & 0.48 & - & & \\
NPP & 0.52 & -0.42 & 0.10 & 0.07 & -0.53 & 0.60 & 0.68 & - & \\
VTM & 0.79 & -0.48 & 0.21 & 0.13 & -0.70 & 0.47 & 0.95 & 0.68 & - \\
QTP & 0.55 & -0.42 & 0.09 & 0.07 & -0.55 & 0.78 & 0.69 & 0.96 & 0.69 \\
\hline
\end{tabular}

Número de potes de mel (NPM), largura dos potes de mel (LPM), altura dos potes de mel (APM), volume dos potes de mel (VPM), número de potes de pólen (NPP), largura dos potes de pólen (LPP), altura dos potes de pólen (APP), volume total do mel (VTM) e quantidade total de pólen (QTP) de 15 colônias de Melipona interrupta.

A partir da quantidade de produção de mel, infere-se que esta esteja correlaciona com a disponibilidade de florada no raio de vôo das abelhas. É possível inferir ainda que houve expressiva participação das operárias campeiras durante o período de coleta de néctar no período de floração na área de estudo. Com esses resultados evidenciam que existe necessidade de 
adotar estratégias de manejo no período de escassez de alimentos, sendo recomendada a alimentação artificial para fortalecimento da colônia para a maximização da produção de mel no período de safra (EVANGELISTARODRIGUES et al., 2008).

Ressalta-se que devem ser consideradas também informações como espécie da abelha, modelo da caixa e tamanho, manejo, ambiente, adaptabilidade e a variabilidade ambiental (KERR, 2006). Todavia, o estudo das correlações para as diferentes espécies, ambiente e manejo são importantes para o conhecimento de produção e reprodução (EVANGELISTARODRIGUES et al., 2008) pois a biometria de produção permite seleção de abelhas (FRAQUINELLO et al., 2013).

Outro fator que deve ser considerado está relacionado ao estresse térmico, pois o mesmo é apontado como um dos principais fatores limitantes da produção animal. Neste sentido, a termografia infravermelho apresenta-se como alternativa de análise de respostas aos fatores ambientais na produção das abelhas. Na tentativa de regular a temperatura nas colmeias, as abelhas reduzem as atividades de coleta de alimentos para produção e reprodução das colônias, tornando-as fracas, inclusive reduzindo até suas funções básicas, podendo ocasionar a morte do enxame (BECKER, 2014).

Segundo Roberto (2014) a termografia infravermelha TIV, é uma tecnologia importante na produção animal, por ser menos invasiva, evitando o estresse, sendo assim uma opção para identificar as condições ambientais e sua influência no comportamento animal, promovendo o diagnóstico das condições destes as determinadas temperaturas. Essa tecnologia contribui no conhecimento sobre a termorregulação em função das alterações térmicas no ninho (KOTRBA et al., 2007).

$\mathrm{Na}$ Figura 4 verifica-se que a temperatura do mel foi maior $\left(37,4^{\circ} \mathrm{C}\right)$ em relação ao do pote $\left(35,5^{\circ} \mathrm{C}\right)$ cuja mínima foi de $34,8^{\circ} \mathrm{C}$. As temperaturas elevadas podem ser explicadas pelo horário de coleta, haja vista que a temperatura aumenta gradativamente 
entre o período que vai das 6h:00min às 15h:00min (CORREIA et al., 2017). Esses autores analisarem colônias de Melipona eburnea em caixas racionais e troncos de árvores.

Na Figura 4 são identificados os padrões térmicos nos alvos imageados, onde é possível identificar os alvos analisados. Na entrada dos potes de mel foram encontrados os valores térmicos mais elevados atingindo os $43,0^{\circ} \mathrm{C}$, com médias de $39,6{ }^{\circ} \mathrm{C}$, sendo o alvo que apresentou a maior amplitude durante as coletas $\left(3,4{ }^{\circ} \mathrm{C}\right)$. Como as abelhas utilizam estratégias de termorregulação observou-se reduzida oscilação da temperatura nos potes de mel, explicada pela capacidade da cera em funcionar como isolante e conservante. A temperatura na parede das caixas e nas áreas internas entre os potes de mel apresentaram valores semelhantes, próximos a $34,0^{\circ} \mathrm{C}$, cuja máxima foi de $38,2^{\circ} \mathrm{C}$, enquanto que a mínima de 33,2 ${ }^{\circ} \mathrm{C}$ foi encontrada nas laterais internas da caixa (Figura 5).

Figura 4. Imagem obtida por termografia infravermelho indicando padrões térmicos em caixas de criação de abelhas na comunidade de Coroca, em Arapiuns, Santarém, Pará.

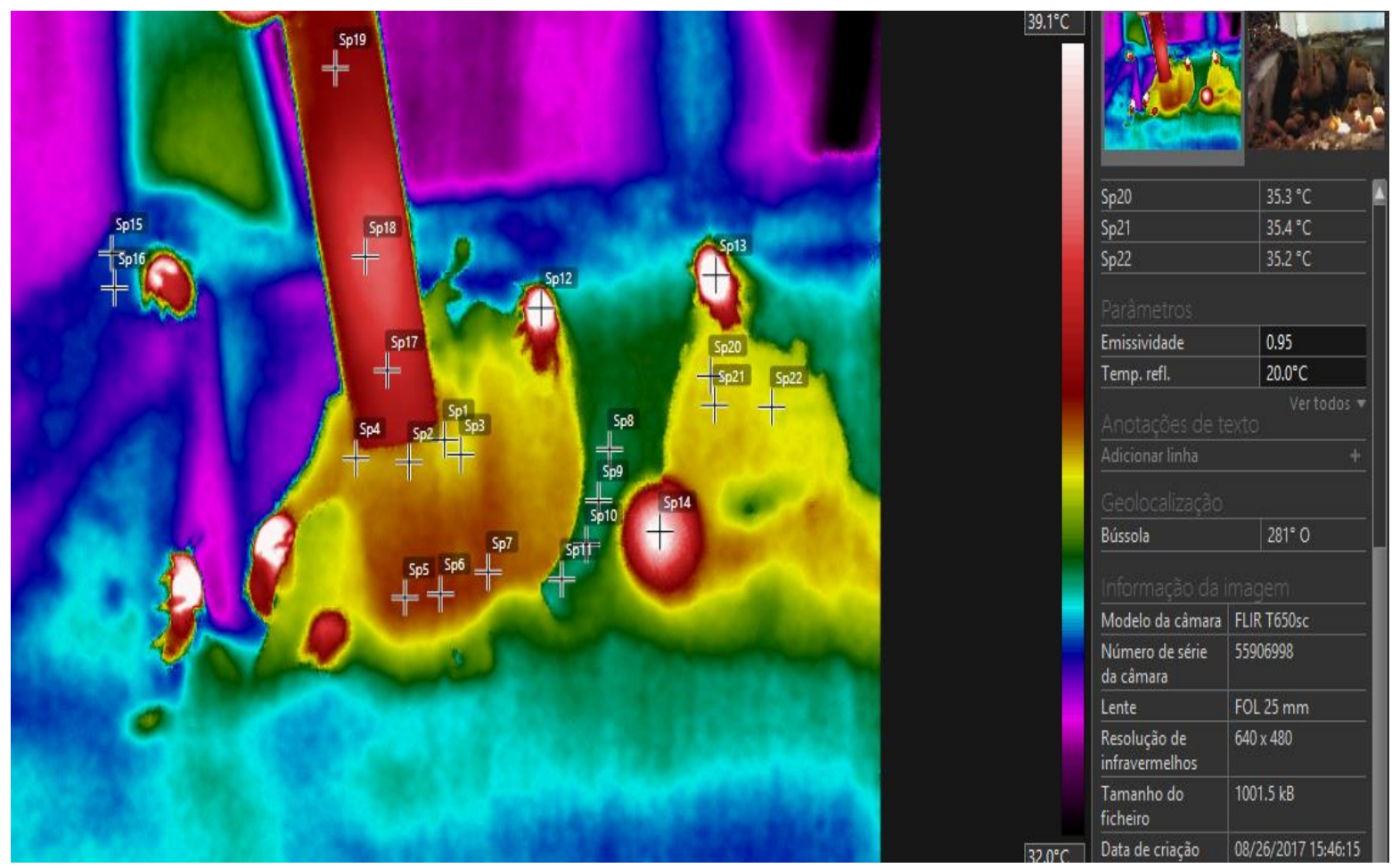


Figura 5. Condições térmicas em alvos dentro de colônias com potes de mel obtidas por termografia infravermelho em criação de abelhas na comunidade de Coroca, em Arapiuns, Santarém.

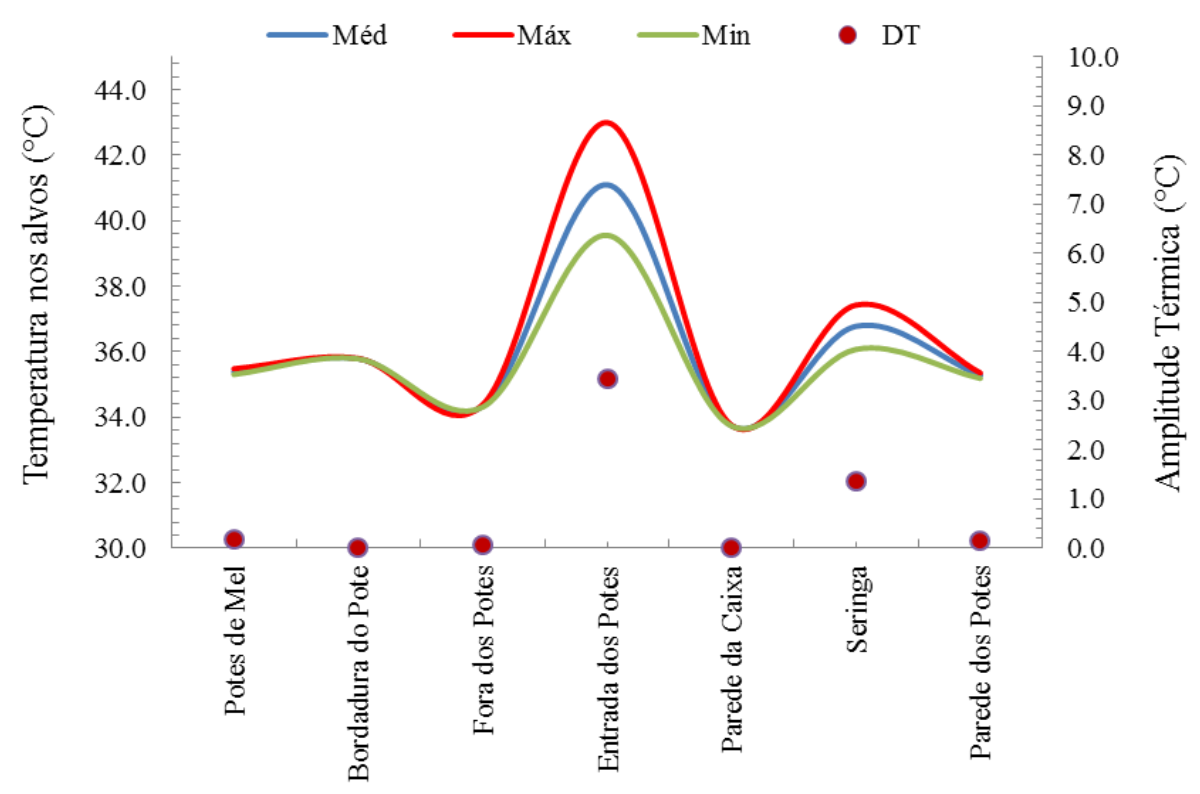

Alvos monitorados nas colmeias

Estudos em diferentes espécies do gênero Melipona apontaram variação na termorregulação (CAMPOS et al., 2010). Roldão (2011) constatou a influência da temperatura ambiente na variação térmica interna em ninhos de Melipona scutellaris. Vollet-Neto (2011) ressalta que a temperatura interna dos ninhos de abelhas nativas muda durante $\mathrm{o}$ ano, mas existe uma faixa térmica específica para sobrevivência das mesmas. Desta forma, as abelhas sociais, usam mecanismos adaptativos, como invólucro, produzido de cera, que envolve a área dos ninhos, os quais formam um isolamento térmico em períodos de baixas temperaturas ambientais.

Mesmo que as abelhas adotem estratégias de termorregulação do ambiente interno, ainda não é o suficiente em condições de temperaturas extremas. De acordo com Becker (2014) as colônias expostas a temperaturas superiores a $34^{\circ} \mathrm{C}$ não conseguem reduzir a temperatura interna para a faixa 
térmica ideal. Também, em período de exposição das colônias a temperaturas inferiores a $26{ }^{\circ} \mathrm{C}$ existe redução nas taxas de sobrevivência com valores que podem chegar a perdas de 60\%. Essas abelhas são mais adaptadas a temperaturas mais elevadas, ou seja, quando submetidas a valores de $32{ }^{\circ} \mathrm{C}$ obtiveram 80\% de sobrevivência. Esses autores explicaram que a espécie $M$. interrupta gasta energia para termorregular o ambiente interno das colônias para controlar a temperatura do microambiente.

\section{CONCLUSÃO}

Em abelhas nativas Melipona Interrupta criadas racionalmente em Arapiuns, Pará, o período de maior produção de mel e pólen ocorre entre julho e dezembro, período menos chuvoso, devendo a produção se concentrar nesses meses, enquanto que nos meses chuvosos deve-se focar em prepara-las para a produção. Em relação à produtividade de mel no período das mais elevadas temperaturas, reforça a adaptabilidade dessa espécie às condições climáticas da região, e a eficiência na termorregulação em ambientes de floresta nativa.

\section{REFERÊNCIAS}

ALVES, R. M. de O. Avaliação de parâmetros biométricos e produtivos para seleção de colônias da abelha uruçu (Melipona scutellaris Latreille, 1811). Bahia, 2010. 104f. Tese (Doutorado em Ciências Agrárias) - Universidade Federal do Recôncavo da Bahia, Brasil. 2010.

BARBOSA-COSTA, K; SANTIAGO, J. M.; FRAXE, T. J. P. Implantação da meliponicultura em comunidade rural de várzea no Amazonas. Cadernos de Agroecologia. v.6, n.2, p.1-6, 2011.

BECKER, T. Desenvolvimento de colmeias de abelhas Melipona interrupta Latreille, 1811 (Hymenoptera: Meliponini) sob diferentes temperaturas em condições de laboratório. Manaus, 2014. 82 f. Dissertação (Mestrado) - Instituto Nacional de Pesquisas da AmazôniaUniversidade Federal do Amazonas (INPA/UFAM), Manaus, Amazonas. 2014.

BROWN, M. J. F.; PAXTON, R. J. The conservation of bees: a global perspective. Apidologie, v.40, n.3, p.410416, 2009.

BUSTOS MAC-LEAN, P.A.; GOSLING, S. N.; BRYCE, E. K.; GABRIEL, K. M. A.; HANES, J.; HONDULA, D. M.; LIANG, L.; MUTHERS, $S_{\text {.; }}$ NASCIMENTO, S. T.; PETRALLI, M.; VANOS, J. A glossary for biometeorology. International Journal of 
Biometeorology (Print), v. 58, p. 277 308, 2014.

CAMARGO, J. M. F.; PEDRO, S. R. M. MELIPONINI LEPELETIER, 1836. IN: MOURE, J.S.; URBAN, D.; MELO, G.A.R. (Orgs). Catalogue of Bees (Hymenoptera, Apoidea) in the Neotropical Region online version, 2012. Disponível em: http://www.moure.cria.org.br/ catalogue. Acesso em 10 Outubro. 2017.

CAMPOS, F. S.; GOIS, G. C.; CARNEIRO, G. G. Colonial thermoregulation in stingless bees (Hymenoptera, Apidae, Meliponini). PUBVET, v.4, n.24, p.1-18, 2010.

CARVALHO-ZILSE，G.A.; NUNES-SILVA, C. G.; ZILSE, N.; SILVA, A. C.; BOAS, H. C. V.; LARAY, J. P.; FREIRE, D. C. B.; KERR, W. E. Criação de abelhas sem ferrão. Iniciativas Promissoras 2: Projeto Manejo dos Recursos Naturais da Várzea. Instituto Brasileiro do Meio Ambiente e Recursos Naturais Renováveis-ProVárzea/IBAMA. Brasília: Edições IBAMA. 27p. 2005.

CARVALHO-ZILSE，G. A.; NUNES-SILVA, C. A. Threats to the stingless bees in the Brazilian Amazon: how to deal with scarce Passos e documentação atualmente necessários para obtenção do Selo de Inspeção Estadual para mel de meliponíneos no estado do Amazonas biological data and na increasing rate of destruction. In: Florio, R.M. (Ed). Bees. Nova Science Publishers, p. 147-168, 2012.

CARVALHO-ZILSE, A. G. Produção de polinizadores para a agricultura na
Amazônia. In: Hiroshi Noda; Luiz Augusto Gomes de Souza; Danilo Fernandes da Silva Filho. (Org.). Pesquisas Agronomicas para a Agricultura Sustentável na Amazônia Central. Manaus. p.19-26, 2013.

CORREIA, F. C. S.; PERUQUETTI, R. C.; FERREIRA, M. G. Termorregulação em colônias de Melipona ebúrnea Friese (Apidae: Meliponina) criadas racionalmente em Rio Branco, Acre. EntomoBrasilis. v.10, n2, p.112-117, 2017.

DIAS, V. H. P.; FILGUEIRA, M. A.; OLIVEIRA, F. L.; DIAS A. M.; COSTA, E. M. Alimentação artificial à base de mel e suas implicações no desenvolvimento de famílias de abelhas jandaíras (Melipona subnitida Ducke) em Mossoró, RN. Revista Verde, v.3, p.40-44, 2008.

EVANGELISTA-RODRIGUES, A.; GÓIS, G. C.; SILVA, C. M.; DARKLÊ, L. S.; SOUZA, D. N.; SILVA, P. C. C.; ALVES E. L.; RODRIGUES, M. L. Desenvolvimento produtivo de colméias de abelhas Melipona scutellaris. Biotemas, v.21, p.5964, 2008.

HEINRICH, B. Hot-blooded insects: Strategies and mechanisms of thermoregulation. Harvard University Press: Cambridge, MA, USA. 1993.

IMPERATRIZ-FONSECA, V. L.; NUNESSILVA, P. As abelhas, os serviços ecossistêmicos e o Código Florestal Brasileiro/Bees, ecosystem services and the Brazilian Forest Code. Biota Neotropica, v. 10, n. 4, p. 59, 2010. 
KERR, W. E.; STORT, A. C.; MONTENEGRO, M. J. Importância de alguns fatores ambientais na determinação das castas do gênero Melipona. Anais da Academia Brasileira de Ciências. v.38, p. 149-168, 1966.

KERR, W. E. Meliponicultura: A importância da meliponicultura para o país. Revista Biotecnologia, Ciência e Desenvolvimento., v.1, n.3, p.42-44, 1997.

KERR, W. E. Método de seleção para melhoramento genético em abelhas. Magistra, v.18, p.209-212, 2006.

KLEIN, A. M.; VAISSIERE, B.; CANE, J. H.; STEFFAN-DEWENTER, I.; CUNNINGHAM, S.A.; KREMEN, C.; TSCHARNTKE, T. Importance of crop pollinators in changing landscapes for world crops. Proc. R. Soc. Lond. B. The Journal of Biological Sciences. v.274, p303-313, 2007.

KOTRBA, R.; KUNC, P.; GÜRDÍL, G. A. K.; PINAR, Y.; SELVÍ, K. C. Aplications of infrared thermography in animal production. Journal of the Faculty of Agriculture. v.22, p.329-336, 2007.

NATES-PARRA, G.; ROSSO-LONDOÑO, J. M. Diversidad De Abejas Sin Aguijón (Hymenoptera: Meliponini) Utilizadas em Meliponicultura Em Colombia. Acta Biológica Colombiana, v.18, n. 3, 2013.

NOGUEIRA-NETO, P. Vida e criação de abelhas indígenas sem ferrão. São Paulo: Editora Nogueirapis, 1997. 446p.

PEREIRA, D. S.; MENEZES, P. R.; BELCHIOR-FILHO, $V_{\text {.; }}$ SOUZA, A. H.;
MARACAJÁ, P.B. Abelhas indígenas criadas no Rio Grande do Norte. Acta Veterinaria Brasilica. v.5, p.81-91, 2011.

ROBERTO, B. V. J; SOUZA, B. B. Utilização da termografia de infravermelho na medicina veterinária e na produção animal. Journal of Animal Behaviour and Biometeorology. v.2, n.3, p.73-84,2014.

ROLDÃO, Y. S. Termorregulação colonial e a influência da temperatura no desenvolvimento da cria em abelhas sem ferrão, Melipona scutellaris (Hymenoptera, Apidade, Meliponini). São Paulo, 2011. 106f. Dissertação (mestrado em entomologia) - Faculdade de Filosofia, Ciências e Letras de Ribeirão Preto, Riberão Preto-USP, 2011.

SAS. User's Guide. SAS Inst., Inc., Cary, NC., 1999.

SOMMER, J. H.; KREFT, H.; KIER, G.; JETZ, W.; MUTKE, J.; BARTHLOTT, W. Projected impacts of climate change on regional capacities for global plant species richness. Proceedings. Biological Sciences / The Royal Society, v.277, n.1692, p.227180, 2010.

SOUSA, B. de A.; CARVALHO, C. A. L. de; ALVES, R. M. de O. Notas sobre a bionomia de Melipona asilvai (Apidae: Meliponini) como subsídio a sua criação racional. Archivos de Zootecnia. v.57, p.53-62, 2008.

SOUZA, D. L.; EVANGELISTARODRIGUES, A.; RIBEIRO, M. N.; PADILLA-ÁLVAREZ, E. S. L. F.; PEREIRA, E. W. E. Análise morfométrica entre Apis mellifera na mesorregião do sertão 
paraibano. Archivos de Zootecnia. v.58, p.65-71, 2009.

VENTURIERI, G. C.; RAIOL, OLIVEIRA, V. F.; PEREIRA, C. A. B. Avaliação da introdução da criação racional de Melipona fasciculata (Apidae: Meliponina), entre os agricultores familiares de Bragança-PA, Brasil. Biota Neotropica, v. 3, n. 2, p. 1-7, 2003.

VOLLET-NETO, A.; MENEZES, C.; IMPERATRIZ-FONSECA, V. L. Brood production increases when artificial heating is provided to colonies of stingless bees. Journal of Apicultural Research. v.50, n.3, 2011. 